\title{
Withania Coagulans in Management of Diabetes Mellitus
}

\author{
Dr. Avinash Shankar \\ MD(Medicine);PGEM(AIIMS), \\ DNB(E\&M)Chairman, \\ National Institute of Health \& ResearchWarisaliganj (Nawada), Bihar. \\ dravinashshankar@gmail.com \\ Dr.Abhishek Shankar, MD \\ AIIMS,Delhi \\ Dr .Shubham, MD (Pediatrics) \\ VMMCH, Delhi. \\ Dr. Amresh Shankar \\ BAMS;MD(Alt.Med),MBA(HA) \\ Bihar State Health Services, Govt of Bihar, Patna.

\section{Dr. Anuradha Shankar, BAMS} \\ National Rural Health Mission \\ Ranchi, Jharkhand
}

\begin{abstract}
Diabetes mellitus, a progressively increasing metabolic and endocrinal disorder and a combined effect of changed diet, life style and generation of non nutrient toxic product in the food and drinks due to increased misuse of fertilizers, chemicals, hormones and adulterants.
\end{abstract}

These days, a disease of comfort and luxury is widely affecting the people who even craving for their lively hood, usually precipitated by alcoholism and stress.

The changed present scenario of diabetes mellitus in the era of advanced diagnostic facilities the patients with >500mg plasma sugar remain conscious and present their history of illness himself whether earlier at this level patient usually became comatose or presents with various consequent sequel.

The most commonly prescribed therapeutic regime remain either Oral hypoglycemic or insulin supplementation which in turn affects the patient's psyche and stress predisposes for rise in blood glucose and its sequel.

Majority prescription used to impose burden rather to modify the continuing diet,clinician usually change the brand, which usually yields with diabetic sequel.

Thus with consideration of bio regulating body metabolism and scavenge free radical from Beta cells of pancreas and increase hepatic utilization of absorbed glucose, an indigenous herb Withania coagulans fruit extract in dose of 5 fruits with warm water morning and evening been assessed and achieved normoglycemic state in 6 weeks of therapy, lipid bio regulation, spared the continuing OHA or Insulin without any adversity and any alteration in hematologic,hepato-renal functions.

These effects are attributed to-

It increases cellular sensitivity, Insulin binding and glucose uptake, scavenges free radicals from Berta cells of pancreas and modulate Insulin secretion improve metabolism,reduces hypothalamic irritation,promote and bioregulate insulin production in pancreas and improve resting energy expenditure.

\section{INTRODUCTION}

Changing dietary status, increasing non dietary constituents in the routinely used food and drinks, and changing life style predisposes for progressively increasing incidence of metabolic and endocrinal disorders. Though available advanced diagnostic methods prompts better research treatment planning, but declining nutritional status predisposes for declined immune protection,rising economical burden 
resulting in mental stress. Thus combination of non dietary constituents and stress load primarily increasing the incidence of diabetes mellitus, its consequent sequel and drug dependency.

In spite of availability of various oral hypoglycemic molecules, non insure bio regulation of blood sugar and check diabetic sequel.

Withania coagulans fruit having established role as hypoglycemic in rat $(1,2)$ been evaluated as an adjuvant with continuing oral hypoglycemic, Insulin and dietary restriction in old cases and dietary restriction only in fresh cases of diabetes mellitus to adjudge its hypoglycemic effect and safety profile.

\section{Materials \& Methods}

Patients of Diabetes mellitus (both old and new)attending centre for metabolic \& endocrinal disease, RA.Hospital \& Research Centre,Warisaliganj (Nawada) were considered for evaluation of Withania coagulans fruit as an adjuvant in bio regulation of blood sugar level in the body, as an adjuvant with continuing anti diabetics in old cases of Diabetes mellitus.

\subsection{Who Criteria for Establishing a Case of Diabetes Mellitus}

Fasting plasma glucose $>7 \mathrm{mmol} / \mathrm{L}$ Or $126 \mathrm{mg} / \mathrm{dl}$

Plasma glucose after 2 hours of 75 gm glucose load : >11.1 mmmol/L Or 200mg/dl

Manifestation of hyperglycemia with random plasma glucose $11.1 \mathrm{mmol} / \mathrm{L}$ Or $200 \mathrm{mg} / \mathrm{dl}$

Glycosylated HbA1C >48

All the selected patients were interrogated for their dietary status,drug therapy and its schedule ,outcome,weight of the patient,blood pressure,diabetic sequel andinvestigated for blood sugar level,both fasting ,PP ,random, urine sugar,lipid profile,hepatic,renal and hematological status along with its neurophysiology. Patients with blood sugar $>500 \mathrm{mg} / \mathrm{dl}$ been also evaluated for their cardiac function with ECG tracing

Each patients were given Withania coagulans fruit watery extract as an adjuvant as -In old cases:

Continue the continuing drug (OHA Or Insulin supplementation Or both) with 5 Withania coagulans fruit watery extract in the early morning and bed time

In freshly detected new cases:

Dietary control with watery extract of 5 fruits of Withania coagulans at the early morning and at bed time

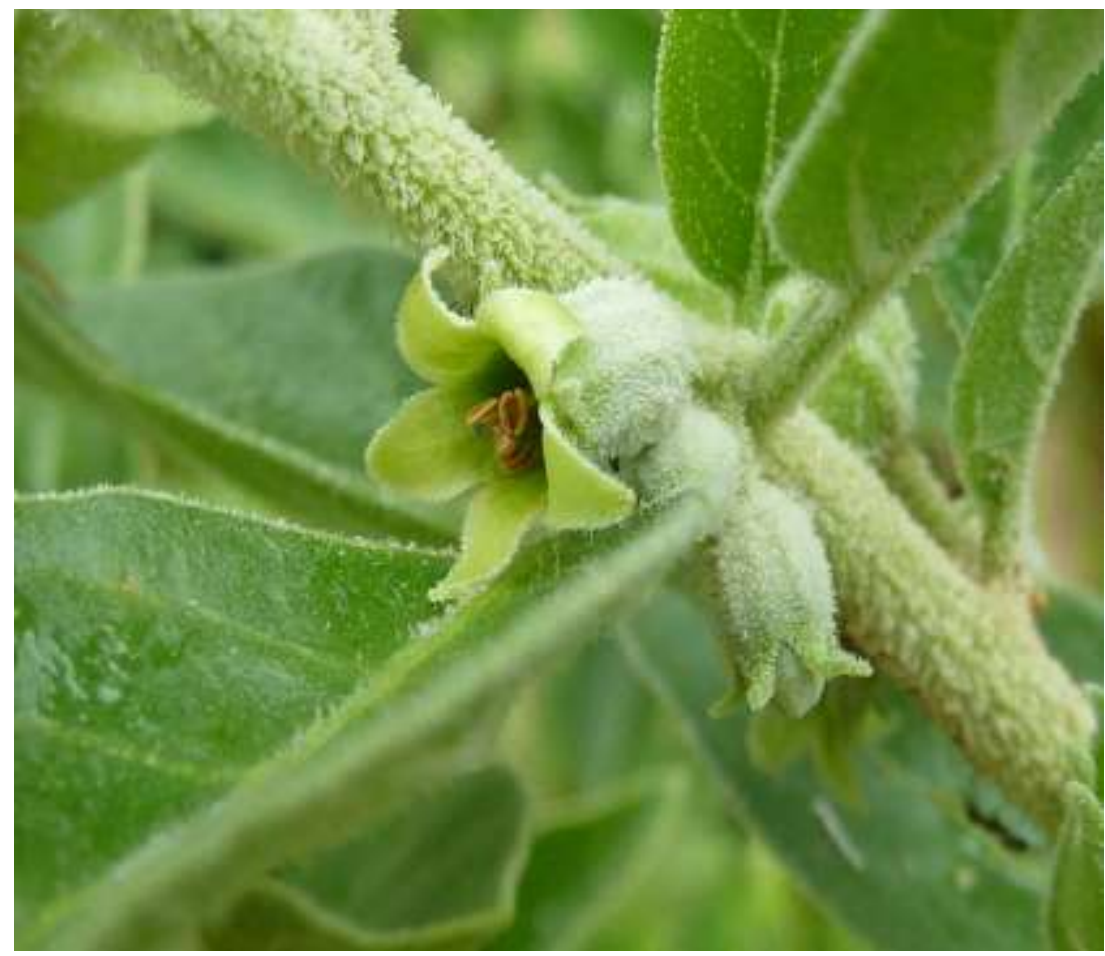




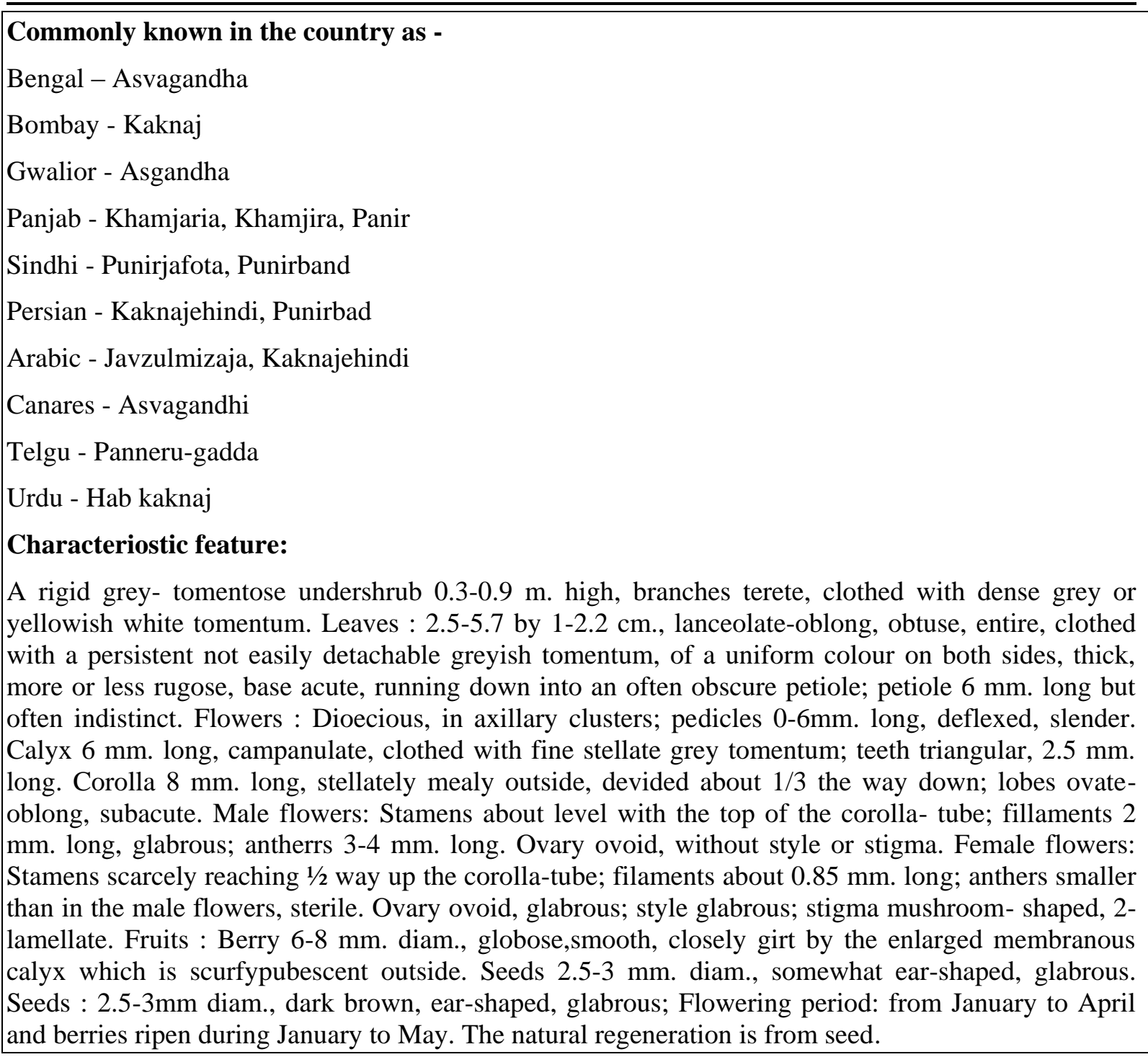

Each selected patients were primarily made aware regarding the treatment modules and taken consent for their inclusion in the proposed study and given a follow up card to asses self-

$>$ Any manifestations suggestive of hypoglycemia

$>$ Self asses urine for the presence of glucose and protein in the urine with Glucostix

$>$ Any manifestation suggestive of allergy or untoward effect of Withania coagulans i.e.Nausea, vomiting,abdominal cramp, lose motion, respiratory discomfort,any other parameters observed.

Dach patients were asked to seek advise on 24 hours help line number for any queries.

On each visit at the centre every $7^{\text {th }}$ day, Blood sugar (both fasting and PP), urine analysis and in old cases having hypoglycemic manifestation or decline in blood sugar level ,continuing anti diabetic drug been gradually tapered down.

In case of Insulin therapy after reducing the Insulin dose, Patients were put on Oral hypoglycemic with adjuvant to maintain the normo glycemic state.

Dietary intake and other physical measures were strictly maintained. To adjudge circadian variationof blood glucose level in patients whose blood sugar (F) >200mg were provided with Glucostix and patients with fasting blood sugar <200mg, Glucometer with strips to repeat the blood sugar assessment before breakfast,lunch and dinner.

Patients hematological, hepatic,renal and lipid profile were repeated on every $30^{\text {th }}$ day to adjudge the effect of adjuvant and asses safety profile Or effect of Withania coagulans on hemato,hepato,renal and lipid profile . 


\section{ObServations}

Among the selected patients $157(40.25 \%)$ were old diabetics non responsive to the continuing anti diabetic drugs and $233(59.75 \%)$ were freshly detected cases of diabetes mellitus

Patients were of age group 30-80 yrs and majority(57.7\%)were of age 40-60 yrs though $12.05 \%$ were of 70-80 yrs,male:female composition of the selected patients was 245:145 while among the old and freshly detected cases were 97:60 and 148:85 respectively.

\section{TABLE \& LEGENDS}

(Table -I , Bar diagram and Pie diagram)

TableI. distribution of patients as per their age, sex and disease status

\begin{tabular}{|c|c|c|c|c|c|}
\hline \multirow{2}{*}{$\begin{array}{c}\text { Age } \\
\text { group }\end{array}$} & \multicolumn{5}{|c|}{ Number of patients } \\
\hline & \multicolumn{2}{|c|}{ Newly detected } & \multicolumn{2}{|c|}{ Old Cases } & Total \\
\hline \multirow[t]{2}{*}{ (in yrs) } & & & & & \\
\hline & Male & Female & Male & Fem & \\
\hline $30-40$ & 20 & 12 & 15 & 08 & 055 \\
\hline $40-50$ & 40 & 20 & 25 & 15 & 100 \\
\hline $50-60$ & 50 & 26 & 30 & 19 & 125 \\
\hline $60-70$ & 20 & 17 & 15 & 11 & 063 \\
\hline 70-80 & 18 & 10 & 12 & 07 & 047 \\
\hline Total & 148 & 85 & 97 & 60 & 390 \\
\hline
\end{tabular}

Selected patients irrespective of their therapeutic status 30(7.6\%) shows blood sugar between 200$250 \mathrm{mg}$ while $10(2.5 \%)$ cases were with blood sugar $>500 \mathrm{mg} \%$,majority $(240 / 61.5 \%)$ cases were with blood sugar 300-400 mg \%. (Table II)

TableII. Bar diagram showing male :female composition

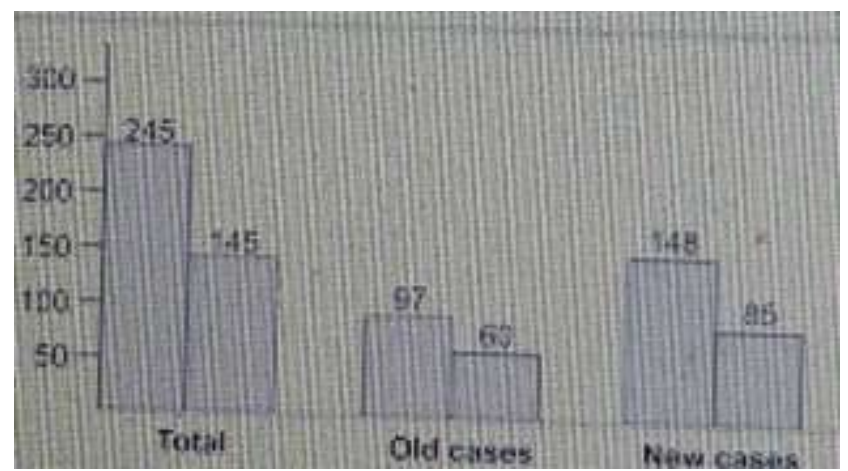

Out of all 84 cases were presenting with hypertension (mean pressure $>120 \mathrm{~mm} \mathrm{Hg}$ ) while rest other were non hypertensive, though among old cases $73(46.5 \%)$ had hypercholesterolemia.(Table III)

TableIII. Pie diagram showing disease wise composition

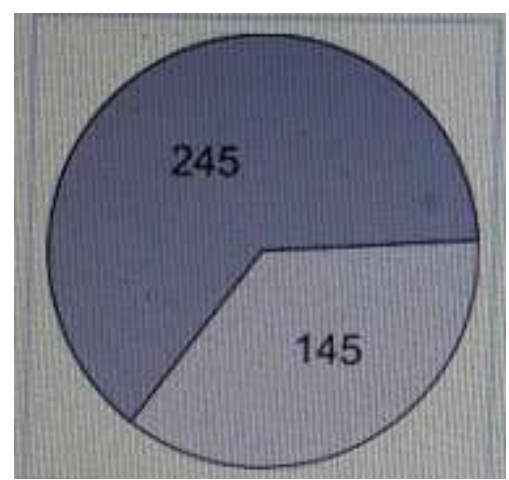

No selected cases had any altered hematological hepatic or renal function alteration, ECG pattern of all the selected cases were within normal limit.

As per dietary status of the patients 164(42\%) and 226(58\%)were taking vegetarian and non vegetarian diet respectively.(Table - Bar diagram ) 
Dr. Avinash Shankar et al.

Among old cases $10(6.3 \%)$ patients were on purely diet restriction while $16(10.1 \%)$ were taking Insulin and 18(11.4\%) combination of multiple hypoglycemic and Insulin,45(28.6\%) cases were on only Metformin and 55 (35\%) on Glipizide .(Table IV)

TableIV. Showing distribution of patients as per their blood sugar level.

\begin{tabular}{|c|c|c|c|}
\hline \multicolumn{2}{|c|}{ Blood Sugar } & Female & Total \\
\hline (in mg\%) & Male & 05 & 30 \\
\hline $200-250$ & 25 & 40 & 90 \\
\hline $250-300$ & 50 & 30 & 80 \\
\hline $300-350$ & 50 & 25 & 70 \\
\hline $350-400$ & 45 & 25 & 65 \\
\hline $400-450$ & 40 & 15 & 45 \\
\hline $450-500$ & 30 & 05 & 10 \\
\hline$>500$ & 05 & & \\
\hline
\end{tabular}

During therapy on $1^{\text {st }}$ week $35(9 \%)$ patients had fasting blood sugar $<100 \mathrm{mg}$ and by $6^{\text {th }}$ week $361(92.6 \%)$ shows fasting blood sugar <100mg.

After $4^{\text {th }}$ week of therapy $388(99.5 \%)$ patients show fasting blood sugar $<120 \mathrm{mg}$ and on $6^{\text {th }}$ week post prandial blood sugar in $337(86.5 \%)<150 \mathrm{mg}$ and in all 390 cases $<160 \mathrm{mg}$.

(Table V and VI)

TableV. Distribution of patients as per average blood pressure

Average blood pressure Number of patients

\begin{tabular}{|c|c|c|c|c|}
\hline & \multicolumn{2}{|c|}{ Fresh (newly detected) } & \multicolumn{2}{|c|}{ Old cases } \\
\hline (in mm Hg) & & - & 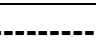 & - \\
\hline & Male & Female & Male & Female \\
\hline$<90$ & $\mathbf{0 2}$ & 01 & 05 & 02 \\
\hline 90-100 & 03 & 01 & 04 & 01 \\
\hline $100-110$ & 77 & 42 & 31 & 23 \\
\hline 110-120 & 42 & 32 & 21 & 18 \\
\hline$>120$ & 24 & 09 & 35 & 16 \\
\hline
\end{tabular}

TableVI. Distribution of old patients as per their therapeutic status

\begin{tabular}{|c|c|c|c|}
\hline \multicolumn{2}{|l|}{ Therapeutic s } & \multicolumn{2}{|c|}{ Number of patients } \\
\hline & Male & Female & Total \\
\hline \multicolumn{4}{|c|}{--} \\
\hline Diet & 06 & 04 & 10 \\
\hline Metformin & 30 & 15 & 45 \\
\hline Glipizide & 36 & 19 & 55 \\
\hline Combination drug & 12 & 06 & 18 \\
\hline Insulin & 07 & 09 & 16 \\
\hline Other oral hypoglycemic & 06 & 07 & 13 \\
\hline
\end{tabular}

In addition majority show decline in serum cholesterol and bio regulate lipid profile. No patients had any hemato,hepato and renal function alteration. (Table VII)

TableVII. Bar diagram showing distribution of patients as per their dietary status

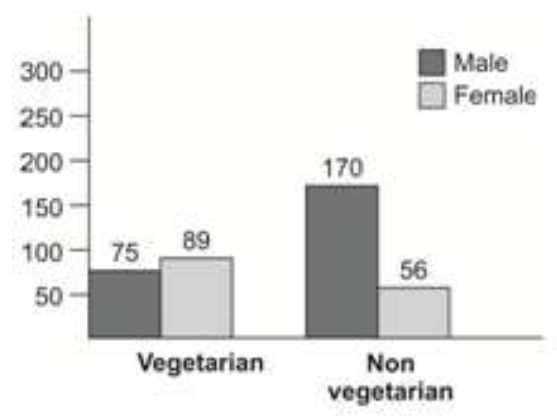




\section{CONCLUSION}

Withania coagulans fruit extract orally early morning and evening at bed time as an adjuvant with continuing anti diabetics in old cases and with OHA in fresh cases progressively established normoglycemic level both fasting and pp and spared the dose of continuing anti diabetic drug and patients taking insulin become completely free of insulin prick.

It also bioregulated lipid profile, alleviated diabetic complication and reduced the dose of OHA without any alteration in hemato, hepatic and renal function.

\section{DisCUSSION}

Diabetes mellitus, a manifestation of altered metabolism is progressively increasing due to declining nutrients in food and drinks, increasing non nutrient constituents of the diet due to progressively increasing use of fertilizers, chemicals, pesticides and adulterants. In addition drugs in vogue i.e.either supplementation of insulin or activate Beta cell secretion, but non bio regulate metabolic function i.e.- metabolism and transformation of glucose which results in circadian variation of blood glucose level.

In the context use of Withania coagulans fruit extract in early morning and at bed time either alone or with continuing OHA or with Insulin bio regulate blood sugar both fasting and PP in 6 weeks in majority cases and also bioregulate lipid profile without any hemato -hepato -renal adversity.

This clinical achievement of Withania coagulans can be explained as $(3,4$ -

Active ingredients of the Withania coagulans fruit extract not only activate Insulin receptor sensitivity but also revitalize Beta cells for Insulin secretion, In addition also bio regulate glucose utilization by hepatic parenchyma i.e.- glucose to glycogen, also bio regulate lipid metabolism,thus Withania coagulans fruit watery extract not only bio regulate glucose utilization, its metabolism and lipid metabolism, maintains HDL/LDL ratio to check diabetic sequel but also -

$>$ makes the cells more sensitive to Insulin binding and glucose uptake,

$>$ improves metabolism by scavenging free radicals in Beta cells for modulated insulin secretion.

$>$ Relieves hypothalamic irritation thus promotes and bio regulate insulin production by pancreas

$>$ Improves resting energy expenditure.

TableVIII. Shows status of fasting blood sugar during therapy

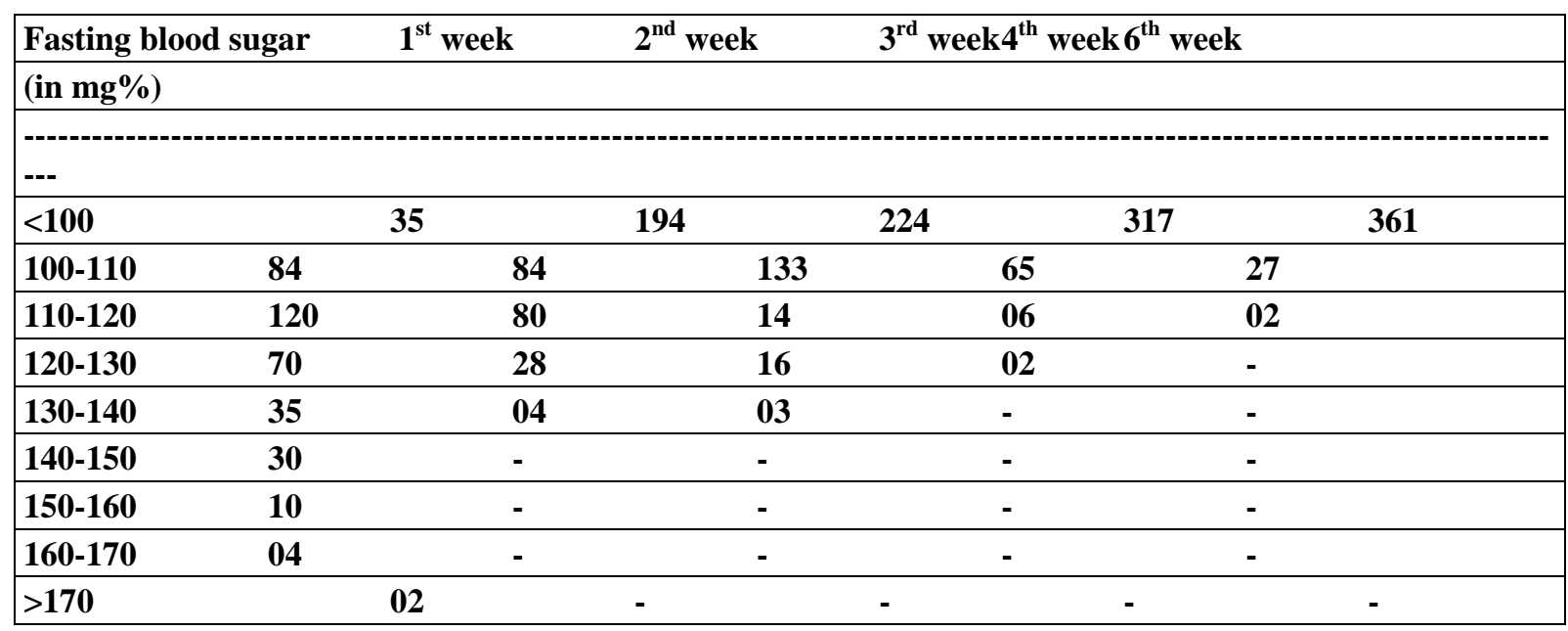

TableIX. Distribution of patients as per post prandial blood sugar during therapy

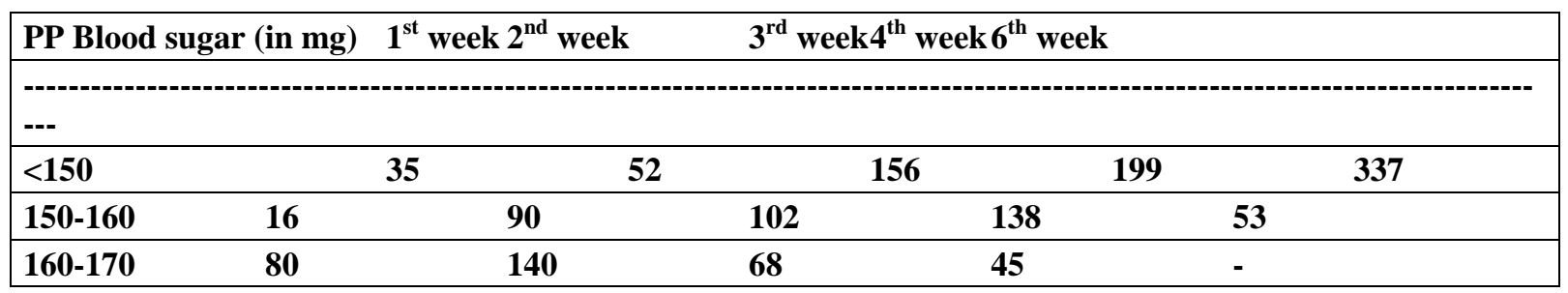


Dr. Avinash Shankar et al.

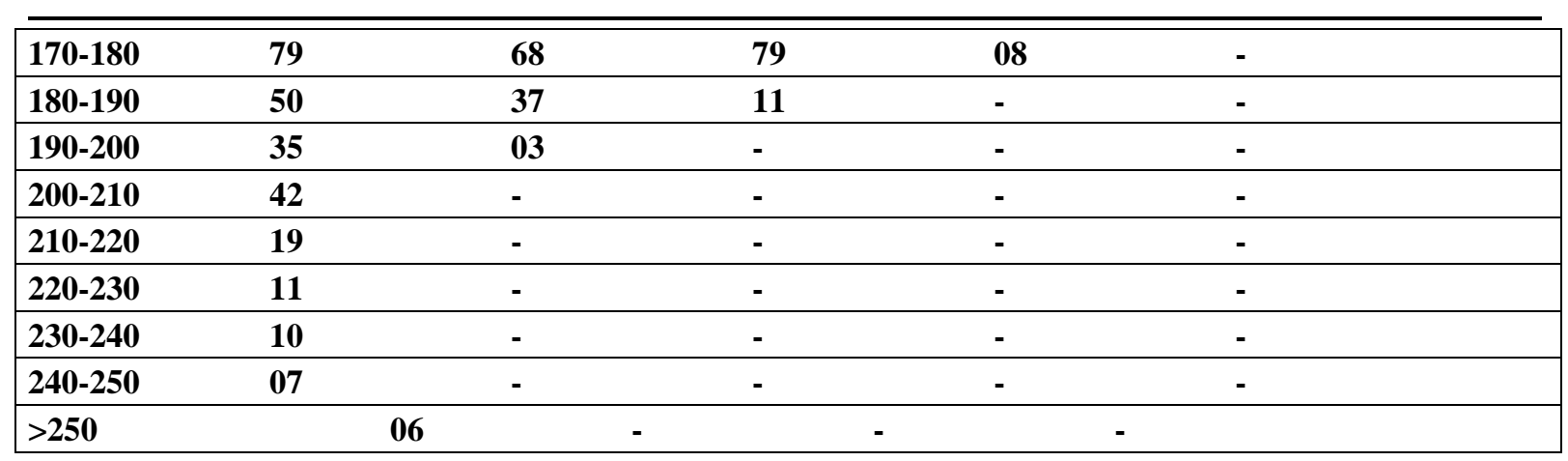

TableX. Distribution of patients as per their basic and post therapy biological values.

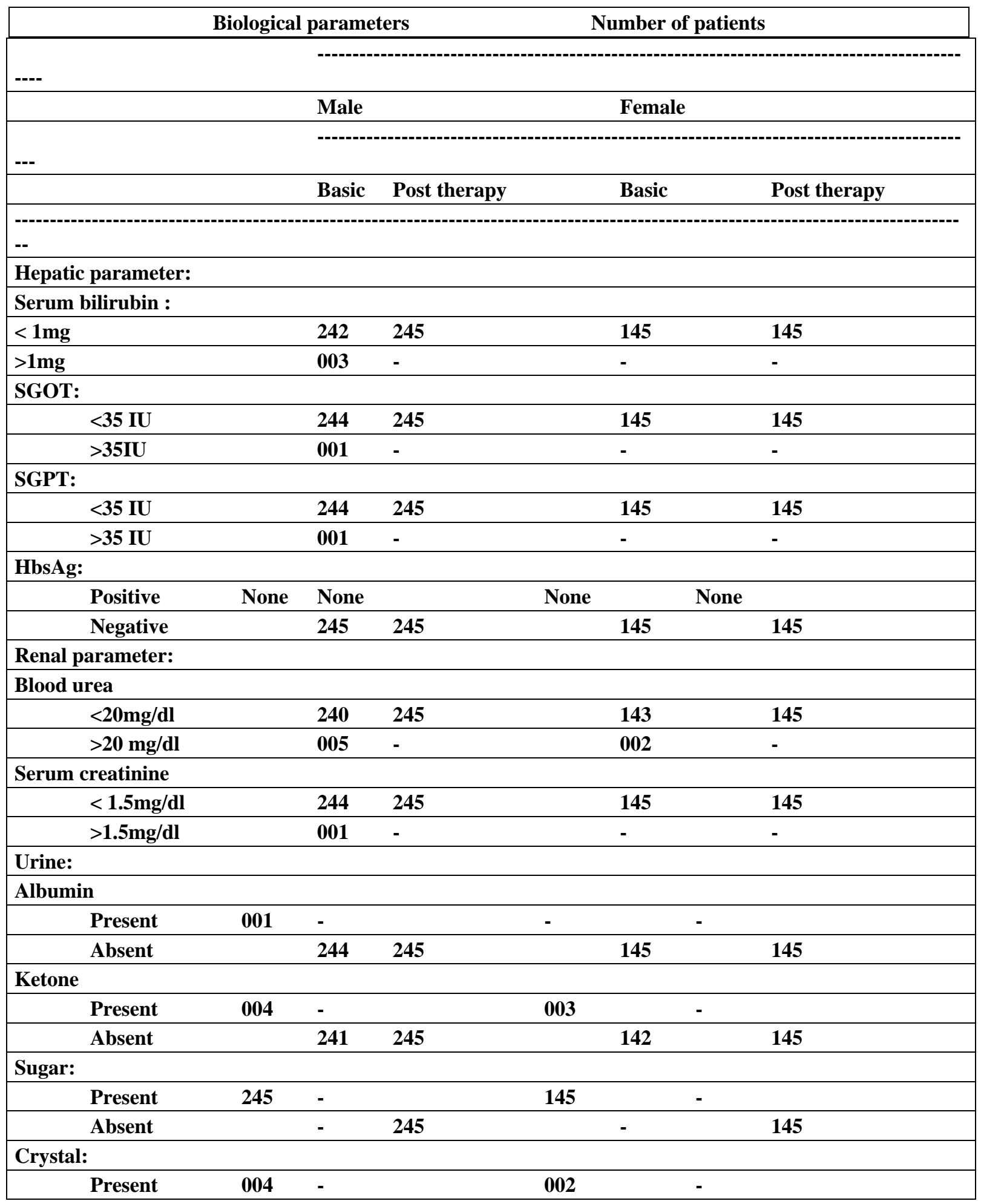


Withania Coagulans in Management of Diabetes Mellitus

\begin{tabular}{|c|c|c|c|c|}
\hline Absent & 241 & 245 & 143 & 145 \\
\hline \multicolumn{5}{|l|}{ Hematological: } \\
\hline \multicolumn{5}{|l|}{ Hemoglobin } \\
\hline$<19 \mathrm{gm} / \mathrm{dl}$ & 195 & - & 138 & - \\
\hline$>10 \mathrm{gm} / \mathrm{dl}$ & 050 & 245 & 007 & 145 \\
\hline \multicolumn{5}{|l|}{ Lipid profile: } \\
\hline \multicolumn{5}{|l|}{ Serum cholesterol } \\
\hline$<200 \mathrm{mg} / \mathrm{dl}$ & 130 & 244 & 080 & 145 \\
\hline $.200 \mathrm{mg} / \mathrm{dl}$ & 115 & 001 & 065 & - \\
\hline \multicolumn{5}{|l|}{ HDL } \\
\hline$<60 \mathrm{mg} / \mathrm{dl}$ & 115 & - & 065 & - \\
\hline$>60 \mathrm{mg} / \mathrm{dl}$ & 130 & 245 & 080 & 145 \\
\hline \multicolumn{5}{|l|}{ LDL } \\
\hline$<60 \mathrm{mg} / \mathrm{dl}$ & 130 & 245 & 080 & 145 \\
\hline$>60 \mathrm{mg} / \mathrm{dl}$ & 115 & - & 065 & - \\
\hline \multicolumn{5}{|l|}{ ECG : } \\
\hline Normal & 245 & 245 & 145 & 145 \\
\hline
\end{tabular}

\section{REFERENCES:}

[1] Mitka, M; Report quantifies Diabetes complication,JAMA,2007/297; 2337-2338

[2] The Diabetes control and complication trial research group, The effects of intensive treatment of diabetes on the development and progress of long term complication in insulin dependent diabetes ,American Diabetic Association.

[3] Standard oc medical care in Diabetes 2009, Diabetes care 2009:32(suppl) (1)

[4] Nathani DM; Clinical practice initial management of glycimia in type II diabetes mellitus, N.Eng.J.Med 2002,347:1342-1349.

[5] Standard of medical care in diabetes mellitus, American Diabetic Asociation 2014,Diabetic care 2014,37(suppl)1;314-317

[6] Diabetes diagnosis and treatment, WHO index for diabetes, WHO bulletin 2014.

[7] Budhiraja,RD;Sudhir,S;Garg,KN Pharmacological investigationof Withania coagulans fruit,Planta medica 32,154-157/1977

[8] Jaishri,D;Ravi,PK;Wattal,G;

Antidiabetic effect of Withania coagulans in experimental rat,Indian journal of Clinical biochemistry,2009/24(1)88-89

[9] Nayar,R;Akanshi Jaynendra,Singh,AD,Shrivastava ,AK;

[10] Coagulonide, a withanolide from Withania coagulans fruit and anti hyperglycemic activity,2008, Bio Organic and medicinal chemistry , 18:6534

[11] Hemlata,S;Nath,AK;Singh,PN,Chansoruria ,JPN,

[12] Hypolipidemic action of aquous extract of Withania coagulans in albino rats, Vol 20,issue: 07,2006/614-617

[13] Shankar ,A; Pharmacological action and plant profile of Withania coagulans Pharmacological basis of Indigenous Therapeutics, Vol.V/Edn 2014 